\title{
C*-IDEALS GENERATED BY POLYNOMIALS
}

\author{
by PAUL TAPPER*
}

(Received 6th May 1997)

\begin{abstract}
The *-algebra $A_{1}$ is defined to be the free unital *-algebra with one generator $x$. A *-ideal $I$ of $A_{1}$ is defined to be a $C^{*}$-ideal if $A_{1} / I$ may be embedded into a $C^{*}$-algebra. It is proved that if $I$ is a *-ideal of $A_{1}$ generated by polynomials in $A_{1}$, then $I$ is a $C^{*}$-ideal. This is not true for general *-ideals of $A_{1}$.
\end{abstract}

1991 Mathematics subject classification: 46L.

\section{Definitions}

Let $W_{1}$ be the set of finite length words in the non-commuting elements $x$ and $x^{*}$. For $w \in W_{1}$, let len(w) denote the length of $w$. Let $A_{1}$ be the free unital involutive algebra over $\mathbb{C}$ generated by the element $x$. So, if $y$ is in $A_{1}$ then $y=\sum_{w \in w_{1}} y_{w} w$, where $y_{w} \in \mathbb{C}$ for all $w$ in $W_{1}$, and only finitely many are non-zero. If $y \in A_{1}$ then call $y$ a *polynomial in $x$. Let $P_{1}$ be the subset of $A_{1}$ which consists of the polynomials in $x$, as opposed to the ${ }^{*}$-polynomials. Say that a word in $W_{1}$ is a syllable if it is of the form $x^{n}$ or $x^{* n}$ for some $n \in \mathbb{N}$.

Given $I \subseteq A_{1}$, say that $I$ is a *-ideal of $A_{1}$ if $I$ is an ideal of $A_{1}$ and is closed under * (the involution on $A_{1}$ ). If $S \subseteq A_{1}$ then let $\langle S\rangle$ denote the ideal of $A_{1}$ generated by $S$ and let $\langle S\rangle_{*}$ denote the *-ideal of $A_{1}$ generated by $S$. So, $\langle S\rangle_{*}=\left\langle S \cup S^{*}\right\rangle$. Say that $I$ is a $C^{*}$-ideal of $A_{1}$ if $I$ is a ${ }^{*}$-ideal of $A_{1}$ and the *-algebra $A_{1} / I$ may be embedded into a $C^{*}$-algebra.

\section{Examples}

The *-ideal $I=\left\langle x^{*} x\right\rangle_{*}$ is not a $C^{*}$-ideal. This is because, if $A_{1} / I$ is embedded in some $C^{*}$-algebra $B$, then, as $x^{*} x \in I$, we have $\left\|x^{*} x\right\|=0$. So, $\|x\|=0$, but $x \notin I$, so $x$ is non-zero in $A_{1} / I$.

It is a result of Goodearl and Menal [2] that $A_{1}$ itself may be embedded into a $\mathrm{C}^{*}$ algebra. So, $\langle 0\rangle_{*}$ is a $C^{*}$-ideal of $A_{1}$. It is a result of Coburn [1] that the *-algebra $A_{1} /\left(x x^{*}-1\right)$. may be faithfully ${ }^{*}$-represented by sending $x$ to the left unilateral shift on $l^{2}(\mathbb{N})$. So, $\left\langle x x^{*}-1\right\rangle$, is a $C^{*}$-ideal of $A_{1}$. There are many other related results in [3].

- Work carried out while supported by a grant from EPSRC. 
For the rest of this paper we shall be interested in the question of whether the * ideals generated by polynomials are $\mathrm{C}^{*}$-ideals.

\section{Definitions}

Let $p$ be a polynomial in $P_{1}$ with $p=\left(x-c_{1}\right) \ldots\left(x-c_{n}\right)$ for some complex numbers $c_{1}, \ldots, c_{n}$ (we may take $p$ to have leading coefficient 1 ). Let $c$ denote the $n$ tuple $\left(c_{1}, \ldots, c_{n}\right)$. Let $I=\langle p\rangle_{*}$. Then, in the quotient *-algebra $A_{1} / I$, we have the identity $x^{n}=x^{n}-p$. As $x^{n}-p$ is a polynomial of degree $n-1$, any element of $A_{1} / I$ may be written as a linear combination of words which have syllables of length at most $n-1$. Whenever considering an element of $A_{1} / I$ we shall assume it is in this form.

For all words $w=x^{r_{R}} x^{* r_{R-1}} \ldots x^{r_{3}} x^{* r_{2}} x^{r_{1}}$ in $A_{1} / I$, (with $r_{j}<n$ for all $j$ ), we can make the following definitions. Let $n_{j}=\sum_{k=1}^{j} r_{k}$ for all $j \geq 1$, and let $H_{w}=l^{2}(\operatorname{len}(w)+1)$ with basis $\left\{\epsilon_{0}, \ldots, \epsilon_{\operatorname{len}(w)}\right\}$. Call $\epsilon_{n_{2}}, \epsilon_{n_{4}}, \ldots$ sources (and also $\epsilon_{0}$ if $n_{1}>0$ ) and call $\epsilon_{n_{1}}, \epsilon_{n_{3}}, \ldots$ sinks. If we were to think of the $\epsilon_{j}$ lined up in order, then any $\epsilon_{j}$ which was not itself a source or a sink would be between a source and a sink. Say that these are the source and sink to which $\epsilon_{j}$ belongs. Still thinking of the $\epsilon_{j}$ as being lined up, let $\delta_{w}(j)$ be (informally) the number of places $\epsilon_{j}$ is from the source it belongs to plus 1 , with a source having a value 1 and a sink having the value for the further of the two sources it is next to. So, if $\epsilon_{j}$ is itself a source then $\delta_{w}(j)=1$, and $\delta_{w}(j+1)=2$, etc. until you go past a sink. For example, if $w=x^{2} x^{* 2} x^{3}$ then the sources are $\epsilon_{0}$ and $\epsilon_{5}$ and the sinks are $\epsilon_{3}$ and $\epsilon_{7}$. If we allow ourselves to write $\delta_{w}$ as acting on tuples of values as well as just single values, then $\delta_{x^{2} x^{* 2} x^{3}}(0,1,2,3,4,5,6,7)=(1,2,3,4,2,1,2,3)$. Note that $\delta_{w}(j) \leq(n-1)+1=n$ for all $j$. by

Define the representation $\mathfrak{I}_{w, c}: A_{1} \rightarrow B\left(H_{w}\right)$ to be the unital *-homomorphism given

$$
\mathfrak{I}_{w, c}(x) \epsilon_{j}= \begin{cases}c_{\delta_{w}(j)} \cdot \epsilon_{j}+\epsilon_{j-1}+\epsilon_{j+1} & \text { if } \epsilon_{j} \text { is a source } \\ c_{\delta_{w}(j)} \cdot \epsilon_{j}+\epsilon_{j+1} & \text { if } 0<j<n_{1}, n_{2}<j<n_{3}, \ldots \\ c_{\delta_{w}(j)} \cdot \epsilon_{j} & \text { if } \epsilon_{j} \text { is a sink } \\ c_{\delta_{w}(j)} \cdot \epsilon_{j}+\epsilon_{j-1} & \text { if } n_{1}<j<n_{2}, n_{3}<j<n_{4}, \ldots\end{cases}
$$

where $\epsilon_{-1}$ and $\epsilon_{\operatorname{len}(w)+1}$ are taken to mean zero.

\section{Examples}

As an example of this definition consider the case when $\mathbf{c}=(1,2,3)$, so $p=(x-1)$ $(x-2)(x-3)$, and $w=x^{2} x^{*} x$. The sources are $\epsilon_{0}$ and $\epsilon_{2}$ and the sinks are $\epsilon_{1}$ and $\epsilon_{4}$. We have $\delta_{x^{2} x^{*} x}(0,1,2,3,4)=(1,2,1,2,3)$ and $\mathfrak{I}_{w, c}(x): \epsilon_{0} \mapsto 1 . \epsilon_{0}+\epsilon_{1}, \quad \epsilon_{1} \mapsto 2 . \epsilon_{1}$, $\epsilon_{2} \mapsto 1 . \epsilon_{2}+\epsilon_{1}+\epsilon_{3}, \epsilon_{3} \mapsto 2 . \epsilon_{3}+\epsilon_{4}, \epsilon_{4} \mapsto 3 . \epsilon_{4}$. 


\section{Theorem}

Theorem. For all words $w$ in $A_{1} / I$ we have $\mathfrak{I}_{w, c}(p)=0$.

Proof. If $\epsilon_{j}$ is a sink then write $p=p^{\prime}\left(x-c_{\delta_{w}(n)}\right)$ where $p^{\prime} \in P_{1}$. Then,

$$
\mathfrak{T}_{w, c}(p) \epsilon_{j}=\mathfrak{I}_{w, c}\left(p^{\prime}\right) \mathfrak{I}_{w, c}\left(x-c_{\delta_{w}(j)}\right) \epsilon_{j}=0 \text {. }
$$

If $\epsilon_{j}$ is neither a sink nor a source, and the sink to which it belongs is $\epsilon_{k}$ where $k>j$ then write $p=p^{\prime}\left(x-c_{\delta_{w}(k)}\right) \ldots\left(x-c_{\delta_{w}(j+1)}\right)\left(x-c_{\delta_{w}(j)}\right)$ where $p^{\prime} \in P_{1}$. Note that $\delta_{w}$ has been defined in such a way that $p$ will not run out of linear factors when writing it in this way. Then,

$$
\begin{aligned}
\mathfrak{T}_{w, \mathrm{c}}(p) \epsilon_{j} & =\mathfrak{I}_{w, \mathrm{c}}\left(p^{\prime}\left(x-c_{\delta_{w}(k)}\right) \ldots\left(x-c_{\delta_{w}(j+1)}\right)\right) \epsilon_{j+1} \\
& =\ldots=\mathfrak{T}_{w, c}\left(p^{\prime}\left(x-c_{\delta_{w}(k)}\right)\right) \epsilon_{k}=0 .
\end{aligned}
$$

Similarly, if $\epsilon_{j}$ is neither a sink nor a source, and the sink to which it belongs is $\epsilon_{k}$ with $k<j$ then $\mathfrak{I}_{w, c}(p) \epsilon_{j}=0$. Finally, if $\epsilon_{j}$ is a source then write $p=p^{\prime}\left(x-c_{\delta_{w}(j)}\right)$ where $p^{\prime} \in P_{1}$. Then,

$$
\mathfrak{I}_{w, c}(p) \epsilon_{j}=\mathfrak{I}_{w, c}\left(p^{\prime}\right) \epsilon_{j-1}+\mathfrak{I}_{w, \mathrm{c}}\left(p^{\prime}\right) \epsilon_{j+1}
$$

By the way we have defined $\delta_{w}$, the polynomial $p^{\prime}$ will still have sufficient linear factors to be able to continue separately as in the two previous cases to get $\mathfrak{I}_{w, c}(p) \epsilon_{j}=0$ as required.

\section{Examples}

To illustrate the previous result let $w=x^{2} x^{* 2} x^{3}$ and $\mathbf{c}=\left(c_{1}, c_{2}, c_{3}, c_{4}\right)$. Consider $\mathfrak{T}_{w, c}(p) \epsilon_{1}$. Write $p=\left(x-c_{1}\right) \cdot\left(x-c_{4}\right)\left(x-c_{3}\right)\left(x-c_{2}\right)$. As

$$
\mathfrak{I}_{w, c}\left(x-c_{2}\right) \epsilon_{1}=\left(c_{2} \epsilon_{1}+\epsilon_{2}\right)-c_{2} \epsilon_{1}=\epsilon_{2}
$$

we have

$$
\mathfrak{T}_{w, c}(p) \epsilon_{1}=\mathfrak{I}_{w, c}\left(\left(x-c_{1}\right) \cdot\left(x-c_{4}\right)\left(x-c_{3}\right)\right) \epsilon_{2}
$$

and continuing in a similar fashion we see that

$$
\mathfrak{I}_{w, c}(p) \epsilon_{1}=\mathfrak{I}_{w, c}\left(\left(x-c_{1}\right)\left(x-c_{4}\right)\right) \epsilon_{3}=\mathfrak{I}_{w, c}\left(x-c_{1}\right) 0=0 .
$$

As another example, consider

$$
\begin{aligned}
\mathfrak{I}_{w, \mathrm{c}}(p) \epsilon_{\mathrm{s}} & =\mathfrak{I}_{w, \mathrm{c}}\left(p^{\prime}\left(x-c_{2}\right)\left(x-c_{1}\right)\right) \epsilon_{\mathrm{s}} \\
& =\mathfrak{I}_{w, \mathrm{c}}\left(p^{\prime}\right)\left(\epsilon_{3}+\epsilon_{7}\right)=\mathfrak{I}_{w, \mathrm{c}}\left(p^{\prime \prime}\left(x-c_{4}\right)\right) \epsilon_{3}+\mathfrak{I}_{w, \mathrm{c}}\left(p^{\prime \prime \prime}\left(x-c_{3}\right)\right) \epsilon_{7}=0 .
\end{aligned}
$$




\section{Corollary}

Corollary. If $p$ is a polynomial in $P_{1}$ then $\langle p\rangle_{*}$ is a $C^{*}$-ideal.

Proof. Take $p=\left(x-c_{1}\right) \ldots\left(x-c_{n}\right)$ along with all the other previous definitions. Firstly, $\mathfrak{I}_{w, c}$ is well-defined on $A_{1} / I$ as $\mathfrak{I}_{w, c}(p)=0$ by Theorem 5 , (where $w$ is a word in $\left.A_{1} / I\right)$. Note that $\left\|\mathfrak{I}_{w, c}(x)\right\| \leq \max \left\{\left|c_{j}\right|\right\}+2$ as $\mathfrak{I}_{w, c}(x)=D+P+Q$ where $D$ is a diagonal operator and $P$ and $Q$ are partial isometries. Therefore, for all $y \in A_{1} / I$, let $v(y)=\sup \left\{\left\|\mathfrak{I}_{w, c}(y)\right\|: w\right.$ a word in $\left.A_{1} / I\right\}$ which is a $\mathrm{C}^{*}$-seminorm on $A_{1} / I$. We are seeking to show that $v$ is a $C^{*}$-norm on $A_{1} / I$. If this is so then we may let $B$ be the $C^{*}$ algebra which is the completion of $A_{1} / I$ with respect to $v$. Then $A_{1} / I$ is embedded in $B$, and we have finished. If $v$ is not a $C^{*}$-norm then there exists some non-zero $y$ in $A_{1} / I$ such that $\mathfrak{I}_{w, \mathrm{c}}(y)=0$ for all $w$ in $A_{1} / I$.

Let $m=\max \left\{\operatorname{len}(v): y_{0} \neq 0\right\}$ and let $w$ be a word of length $m$ with $y_{w} \neq 0$. Let $\epsilon=\epsilon_{0}$ and $\epsilon^{\prime}=\epsilon_{m}$. Given $\alpha$ in $H_{w}$, let $d(\alpha)=\max \left\{j:\left\langle\epsilon_{j}, \alpha\right\rangle \neq 0\right\}$. Informally, this represents the distance along the basis that $\alpha$ contains information. Write $\mathfrak{I}_{w, c}(x)=t$. Considering the action of $t$ on $\alpha$ in $H_{w}$ we see that both $t$ and $t^{*}$ can only move information along to the right by at most one basis vector or, more formally, $d(t \alpha) \leq d(\alpha)+1$ and $d\left(t^{*} \alpha\right) \leq d(\alpha)+1$. If $u$ is a word then $d(u(t) \epsilon) \leq \operatorname{len}(u)$ with equality only being attained if each letter of the word $u$ increases $d$. The *-representation $\mathfrak{I}_{w, c}$ is defined in such a way that $d\left(\mathfrak{I}_{w, c}(w) \epsilon\right)=m$. Let $v$ be a word other than $w$. If $y_{v}=0$ then clearly $\left\langle y_{v} \mathfrak{I}_{w, c}(v) \epsilon, \epsilon^{\prime}\right\rangle=0$. If $y_{v} \neq 0$ then either $\operatorname{len}(v)<m$, in which case $d\left(\mathfrak{I}_{w, c}(v) \epsilon\right)<m$, or $\operatorname{len}(v)=m$. It is not hard to see that if $\operatorname{len}(v)=m$ and $v \neq w$ then we again have $d\left(\mathfrak{I}_{w, c}(v) \epsilon\right)<m$ (informally, in this case the operator turns back, or stops, at some point along the basis). Thus, $\left\langle\mathfrak{I}_{w, c}(y) \epsilon, \epsilon^{\prime}\right\rangle=y_{w}\left\langle\mathfrak{I}_{w, c}(w) \epsilon, \epsilon^{\prime}\right\rangle \neq 0$, and $\mathfrak{I}_{w, c}(y) \neq 0$ as required.

Note that, for the particular case where $p(x)=x^{n}$ and $n \in\{1,2,3, \ldots\}$, we could replace the operator $\mathfrak{I}_{w, c}$ with $\lambda$. $\mathfrak{I}_{w, c}$, where $\lambda$ is any positive number. This would give us the stronger result that, in this case, $A_{1} /\langle p\rangle_{*}$ can be embedded into a $\mathrm{C}^{*}$-algebra so that $\|x\|=M$ for any positive real $M$.

If $y \in A_{1}$ and $m$ is the maximum length of a word with non-zero coefficient in $y$, then taking $p(x)=x^{m+1}$, we get a ${ }^{*}$-representation $\pi$ of $A_{1}$ such that $\pi(y) \neq 0$. This implies the result of Goodearl and Menal referred to in Examples 2.

\section{Corollary}

Corollary. If $p_{1}, \ldots, p_{r}$ are in $P_{1}$ then $\left\langle p_{1}, \ldots, p_{r}\right\rangle_{*}$ is a $C^{*}$-ideal.

Proof. By elementary algebra we know that there exists some polynomial $q$ such that $\left\langle p_{1}, \ldots, p_{r}\right\rangle_{*}=\langle q\rangle_{*}$. By Corollary 7 this is a $C^{*}$-ideal.

Thus, if $I$ is any *-ideal in $A_{1}$ which is generated by polynomials then $I$ is a $C^{*}$-ideal of $A_{1}$ and $A_{1} / I$ may be embedded into a $C^{*}$-algebra. 


\section{REFERENCES}

1. L. A. Coburn, The $\mathrm{C}^{*}$-algebra generated by an isometry, Bull. Amer. Math. Soc. 73 (1967), 722-726.

2. K. R. Goodearl and $P$. Menal, Free and residually finite-dimensional $C^{*}$-algebras, $J$. Funct. Anal. 90 (1990), 391-410.

3. P. N. TAPPER, Embedding *-algebras into $\mathrm{C}^{*}$-algebras and $\mathrm{C}^{*}$-ideals generated by words, J. Operator Theory, submitted.

DepartMent OF PURE MATHEMATICS

LEEDS UNIVERSITY

LEEDS, LS2 9JT

UNITED KINGDOM

E-mail address: paul_tapper@hotmail.com 